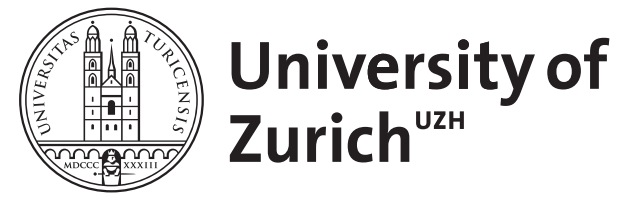

\title{
Jugend schützt nicht vor Schlaganfall: Was die hormonelle Verhütung zum
} Risiko beiträgt

Djalali, S

DOI: https://doi.org/10.1024/1661-8157/a001093

Other titles: Youth does not protect against stroke: what hormonal contraception contributes to risk

Posted at the Zurich Open Repository and Archive, University of Zurich

ZORA URL: https://doi.org/10.5167/uzh-73569

Journal Article

Accepted Version

Originally published at:

Djalali, S (2012). Jugend schützt nicht vor Schlaganfall: Was die hormonelle Verhütung zum Risiko beiträgt. Praxis, 101(22):1445-1446.

DOI: https://doi.org/10.1024/1661-8157/a001093 


\section{Jugend schützt nicht vor Schlaganfall: Was die hormonelle Verhütung zum Risiko beiträgt}

Frage:

Lassen moderne hormonelle Verhütungsmethoden nicht nur das Risiko für venöse Thromboembolien ansteigen, sondern auch für arterielle Infarkte?

\section{Hintergrund:}

Dass hormonelle Verhütungsmethoden mit einem Risiko für venöse Thromboembolien assoziiert sind, wurde oft untersucht und belegt. Seltener geraten arterielle Geschehen in den Blickpunkt, sollten bei einer umfänglichen Verhütungsberatung jedoch ebenfalls abgewogen werden. Die Studienlage diesbezüglich war bislang widersprüchlich. In dieser Studie wird nun gezielt das Auftreten von zerebralen Infarkten und Myokardinfarkten bei jungen Frauen untersucht, die mit oralen Kontrazeptiva resp. IUDs, Vaginalringen, subkutanen Implantaten oder östrogenhaltigen Pflastern verhüten.

\section{Einschlusskriterien:}

- Frauen zwischen 14 und 49 Jahren, deren Gesundheitsdaten (Medikamentenverschreibungen, Austrittsdiagnosen nach Spitalaufenthalt resp. Todesursache) für den Zeitraum 1995-2009 in dänischen Gesundheitsregistern verfügbar waren.

\section{Ausschlusskriterien:}

- Arterielle und/oder venöse Thrombosen in der Vorgeschichte

- Gerinnungsstörungen

- Malignom

- Sterilisation

- Temporärer Ausschluss bei Schwangerschaft; Wiedereinschluss drei Monate post partem

\section{Studiendesign und Methode:}

Offene Kohortenstudie auf Basis von Registerdaten.

\section{Studienort:}

Dänemark

\section{Intervention:}

- keine

\section{Outcome:}

- Primärer Endpunkt: Auftreten eines thromboembolischen Schlaganfalls oder eines Myokardinfarkts

\section{Resultat:}

- Die Daten von 1'626'158 Frauen wurden in die Analyse eingeschlossen. Dies entsprach 4,9 Mio. Personenjahren der Anwendung hormoneller Verhütungsmethoden resp. 9,3 Mio. Personenjahren ohne hormonelle Verhütung. 
- Unabhängig von Alter, Komorbiditäten, Dauer der Hormoneinnahme und Gestagentyp, war das Risiko eines thromboembolischen Schlaganfalls resp. einen Myokardinfarkts am grössten bei Verwendung von Kombinationspräparaten mit hoher Östrogendosis.

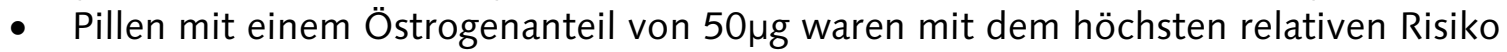
assoziiert (1,97 für Schlaganfall; 3,73 für Myokardinfarkt) gegenüber Pillen mit einem Östrogenanteil zwischen 30 und $40 \mu \mathrm{g}$ (1,75 für Schlaganfall; 1,88 für Myokardinfarkt) bzw. $20 \mu \mathrm{g}$ (1,6 für Schlaganfall; 1,4 für Myokardinfarkt).

- Zwischen den verschiedenen Kombinationsprodukten mit unterschiedlichen Gestagenkomponenten liessen sich keine statistisch signifikanten Risikounterschiede feststellen.

- Für reine Gestagenpräparate wie das Levonorgestrel-Intrauterinpessar (IUP) oder subkutane Implantate war das Schlaganfallrisiko verringert (IUP: 0,73; Implantat: 0,88) während das Myokardinfarktrisiko erhöht war (IUP: 1,02; Implantat: 2,14).

- Für Vaginalring und Östrogenpflaster war das Schlaganfallrisiko erhöht (Ring: 3,15; Pflaster: 2,49), bezüglich Myokardinfarkten war die Fallzahl zu klein für eine valide Datenauswertung.

- Das Risiko für Frauen nach Beenden der hormonellen Verhütung war gleich hoch wie bei Frauen, die keine hormonelle Hütung benutzten.

\section{Kommentar:}

- Arterielle thromboembolische Ereignisse nehmen vor allem im Alter zu. Das Grundrisiko einer jungen Frau im gebärfähigen Alter ein solches Ereignis zu erleiden, ist prinzipiell als gering einzuschätzen. Venöse Thrombosen treten ungleich häufiger auf und können durch eine hormonelle Verhütungsmethode begünstigt werden. Gleichwohl legen die Ergebnisse dieser Studie nahe, dass auch das Risiko für arterielle thrombotische Verschlüsse durch die Einnahme einer hormonellen Kontrazeption durchaus erhöht wird und bei der Verhütungsberatung zusammen mit den übrigen Risikofaktoren individuell in Betracht gezogen werden sollte.

- Aus praktischer Sicht ist vor allem das Studienergebnis bemerkenswert, dass niedrigdosiertes Östrogen das Risiko offenbar weniger stark erhöht als hochdosiertes Östrogen. Dies bestätigt den allgemeinen Trend: Pillen mit $50 \mu \mathrm{g}$ Östrogen sind in den letzten Jahren selten geworden, zunehmend werden Präparate mit 20-30 $\mu \mathrm{g}$ verschrieben.

\section{Literatur:}

Lidegaard $\varnothing$, Løkkegaard E, et al.: Thrombotic Stroke and Myocardial Infarction with Hormonal Contraception. N Engl J Med 2012; 366: 2257-2266.

\section{Verfasser:}

Dr. med. Sima Djalali 